
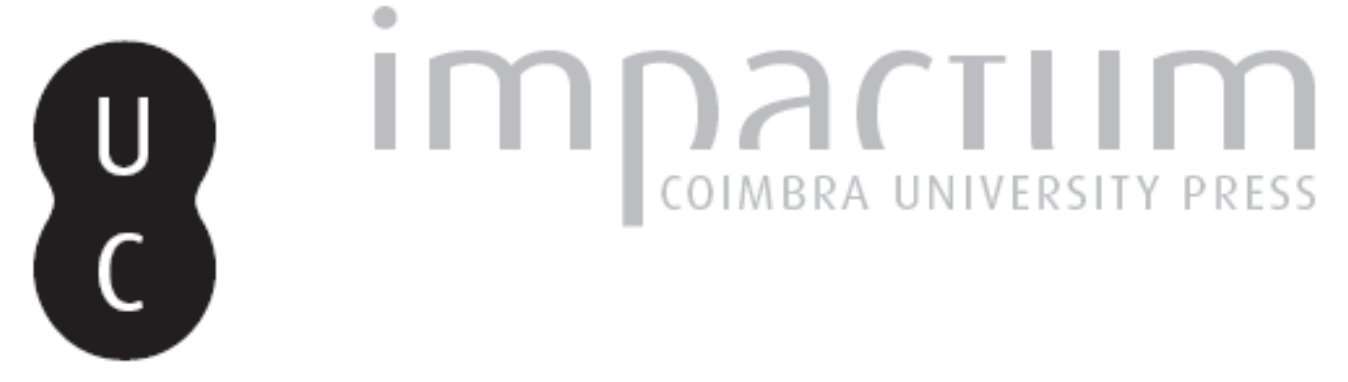

\title{
Abuso contra crianças: a perceção do médico na intervenção pediátrica
}

Autor(es): $\quad$ Trovão, J. Nuno; Frazão, S.; Magalhães, T.

Publicado por: Imprensa da Universidade de Coimbra

URL persistente:

URI:http://hdl.handle.net/10316.2/33266

DOI:

DOI:http://dx.doi.org/10.14195/1647-8630_23_8

Accessed : $\quad$ 26-Apr-2023 16:05:42

A navegação consulta e descarregamento dos títulos inseridos nas Bibliotecas Digitais UC Digitalis, UC Pombalina e UC Impactum, pressupõem a aceitação plena e sem reservas dos Termos e Condições de Uso destas Bibliotecas Digitais, disponíveis em https://digitalis.uc.pt/pt-pt/termos.

Conforme exposto nos referidos Termos e Condições de Uso, o descarregamento de títulos de acesso restrito requer uma licença válida de autorização devendo o utilizador aceder ao(s) documento(s) a partir de um endereço de IP da instituição detentora da supramencionada licença.

Ao utilizador é apenas permitido o descarregamento para uso pessoal, pelo que o emprego do(s) título(s) descarregado(s) para outro fim, designadamente comercial, carece de autorização do respetivo autor ou editor da obra.

Na medida em que todas as obras da UC Digitalis se encontram protegidas pelo Código do Direito de Autor e Direitos Conexos e demais legislação aplicável, toda a cópia, parcial ou total, deste documento, nos casos em que é legalmente admitida, deverá conter ou fazer-se acompanhar por este aviso.

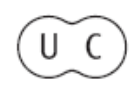


REVISTA PORTUGUESA

$\stackrel{\infty}{\cdots}$

$\stackrel{2}{i}$

$\dot{\bar{x}}$

$\bar{x}_{\bar{x}}$

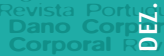

Oeno dorporal Revista Portuguesta do Dano Corporal Revista Portuguens do Dano Corporal

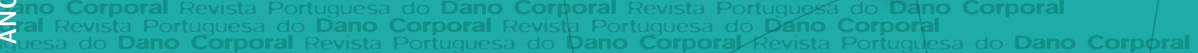

Rvista Portuguesa do Dano Corporal Revista Portuguesa do Dano Corporal Revista Portuguesa do pang Coporal

NPortuguesa do Dano Corporal Revista Portuguesd do Dano Corporal

Silo

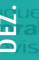

\section{APADAC}

ASSOCIAÇÃO PORTUGUESA

DE AVALIAÇÃO

DO DANO CORPORAL

INSTITUTO NACIONAL

DE MEDICINA LEGAL, I.P.

DELEGAÇÃO DO CENTRO 


\title{
Abuso contra crianças. A perceção do médico na intervenção pediátrica
}

\author{
J. Nuno Trovão(1), S. Frazão(1,2,3), T. Magalhães ${ }^{(1,2,3,4)}$
}

\section{INTRODUÇÃO}

O abuso, onde se inclui a violência doméstica e os maus tratos, constitui, além de uma questão legal e social, um problema grave e frequente de saúde, com consequências sempre severas, que podem até ser fatais. Assim, é atualmente considerado uma patologia, constante em vários capítulos da Classificação Internacional de Doenças da Organização Mundial de Saúde (CID-10). O abuso é particularmente grave no caso da violência doméstica, que se pode definir como qualquer forma de comportamento físico e/ou emocional, não acidental e inadequado, resultante de disfunções e/ou carências nas relações interpessoais, num contexto de uma relação de dependência por parte da vítima e de confiança e poder por parte do abusador que, habitando, ou não, no mesmo agregado familiar, seja cônjuge ou ex-cônjuge, filho/a ou outro familiar1. Mas outras situações podem ser também graves, ainda que perpetradas noutros contextos, designadamente em contexto institucional, importando referir, entre outros, os casos de bullying.

Estes casos são particularmente complexos, delicados e graves quando perpetrados contra crianças, dada a dificuldade de os detetar. No caso das crianças até aos 3 anos, pelo menos, estas não têm capacidade nem para perceber o abuso nem para o revelar, sendo que muitas ficam em casa e não frequentam o infantário, pelo que frequentemente o único olhar externo que

\footnotetext{
Instituto de Ciências Biomédicas Abel Salazar da Universidade do Porto

Instituto Nacional de Medicina Legal e Ciências Forenses, I.P. - Delegação do Norte

Centro de Ciências Forenses - CENCIFOR, Portugal

4 Faculdade de Medicina da Universidade do Porto, Departamento de Medicina Legal e Ciências Forenses
} 
sobre elas existe é o do médico pediatra. No caso das crianças mais velhas, existe, tal como em todos os casos das vítimas deste tipo de violência, dificuldade para revelar o abuso, seja porque não entendam certos comportamentos como abusivos (e.g., o castigo físico para educação), seja por vergonha ou medo.

Assim, nestas situações, o papel dos serviços de saúde, designadamente ao nível da Pediatria, revela-se fundamental na sua deteção, sinalização e/ou denúncia, para além do papel ao nível do seu tratamento e acompanhamento. Se a intervenção dos profissionais que atuam nesta primeira linha de deteção não for correta, todas os outros níveis de intervenção, visando o diagnóstico médico-legal, a proteção e tratamento da criança, bem como a sua reabilitação e da respetiva família e, ainda, em certos casos, a investigação criminal, não se concretizarão com sucesso.

$\mathrm{O}$ abuso pode manifestar-se através de diversas formas. As mais conhecidas são:

a) Abuso físico: qualquer comportamento intencional, recorrente ao uso da força (incluindo castigos corporais) que possa provocar dano físico ${ }^{2}$. São sobretudo suspeitas as lesões cujo mecanismo de produção não é coerentemente explicado, moduladas, em diferentes estadios de evolução, em locais corporais impróprios para acidentes e quando há atraso na procura de cuidados de saúde;

b) Abuso psicológico ou emocional: ausência intencional de suporte afetivo, englobando ameaças e depreciação permanente ${ }^{3}$. Os indícios principais são, entre outros, a aversão ao contato físico, extrema obediência, apatia, agressividade, mau aproveitamento escolar e perturbações de ansiedade, depressivas, da fala, do sono e do comportamento alimentar;

c) Abuso sexual: envolvimento da criança em práticas que visam a gratificação sexual do abusador, as quais ela não tem capacidade para compreender ou dar o seu consentimento ${ }^{5}$. A sua deteção e diagnóstico são particularmente difíceis, devido ao tabu social, à ausência de testemunhas oculares e à dificuldade em encontrar vestígios físicos ou biológicos, bem como lesões (sobretudo quando os casos são revelados tardiamente). Acresce que as lesões genitais e os sintomas geralmente não são específicos, sendo aqui fundamental a precocidade da realização do exame médico-legal ${ }^{5}$.

Entre outras práticas abusivas, pode ainda referir-se a exposição da criança a um contexto familiar de violência nas relações de intimidade ${ }^{6,7}$, a 
síndrome de Munchausen por Procuração e a exploração pela mendicidade, pelo trabalho ou pela prostituição ${ }^{1}$.

Em Portugal estes comportamentos abusivos assumiram em 2001 a natureza de crime público, sendo a intervenção nestes casos da responsabilidade do Ministério Público (MP), independentemente da vontade da vítima ou do seu representante legal. A denúncia nos crimes de natureza pública é "obrigatória, ainda que os agentes do crime não sejam conhecidos, para os funcionários (...) quanto a crimes de que tomaram conhecimento no exercício das suas funções e por causa delas" (artigo $242^{\circ}$ do Código do Processo Penal). Tratando-se de vítimas de menor idade, a obrigação de comunicação dos casos em que a criança possa estar em perigo é alargada à população geral, de acordo com o artigo $66^{\circ}$ da Lei 147/99, de 1 de setembro, o qual consagra a promoção e proteção da criança ou adolescente em risco. A comunicação destes casos é também obrigatória para os médicos, à luz do Código Deontológico da Ordem dos Médicos Portuguesa.

A denúncia consiste na comunicação formal do crime ao MP, a qual pode ser feita por qualquer pessoa ou entidade, diretamente ou por intermédio dos órgãos de polícia criminal ou dos médicos do Instituto Nacional de Medicina Legal e Ciências Forenses, I.P. (INMLCF), sendo o objetivo promover a investigação criminal, a qual também pode ser um processo chave para a proteção da vítima. Paralelamente a esta, e no caso das crianças, deve ser feita a sinalização da suspeita às entidades com competência em matéria de infância e juventude da área de residência da criança, neste caso com o objetivo da sua promoção e proteção, designadamente à Comissão de Proteção de Crianças e Jovens (CPCJ) da área de residência da criança.

No caso da existência de lesões, ou suspeitando-se da possibilidade da sua existência, é obrigatória, por lei, a realização de uma perícia médico-legal, devendo tal procedimento, sobretudo nos casos de abuso sexual, reger-se pelo protocolo celebrado em 2011 entre os Ministérios da Saúde e da Justiça, e a Comissão Nacional de Proteção de Crianças e Jovens ${ }^{8}$.

\section{OBJETIVO}

Este estudo teve como objetivo geral analisar as perceções dos médicos que trabalham na área da Pediatria, relativamente à sua capacidade para intervirem nesta matéria. Especificamente pretendeu-se saber se estes médicos têm consciência da dimensão dos comportamentos abusivos a que são sujeitas certas crianças, se se sentem aptos a detetar os indicadores de abuso, se conhecem e se efetivamente cumprem com os procedimentos de sinalização e denúncia previstos para estes casos e a necessidade sentida de formação ou de implementação de protocolos de atuação. 


\section{MATERIAL E MÉTODOS}

Elaborou-se um questionário de autoavaliação, de preenchimento anónimo, dirigido aos vários pediatras e internos de Pediatria a prestar serviço nos hospitais da área de influência da Administração Regional de Saúde do Norte (ARS). O questionário foi enviado por correio ou por via eletrónica, após contato telefónico com os diretores de serviço de Pediatria e após a obtenção de autorização pelos Conselhos de Administração (CA) dos respetivos hospitais. Neste questionário de escolha múltipla analisaram-se os seguintes pontos: (a) a noção da responsabilidade de detetar casos de abuso; (b) a aptidão sentida para detetar os indicadores de abuso; (c) o conhecimento sobre os procedimentos de sinalização e denúncia dos casos suspeitos e sobre a diferença entre ambos; (d) o número de vítimas detetadas e a sinalização e/ou denúncia desses casos; (e) a articulação com os Núcleos Hospitalares de Apoio a Crianças e Jovens em Risco (NHACJR) e com o "Médico de Família"; (f) a noção da responsabilidade de sinalizar e da obrigatoriedade legal de denunciar; (g) a necessidade sentida relativamente à necessidade de implementação de protocolos de intervenção nestes casos.

Dos 15 hospitais contatados, obteve-se resposta positiva dos CA (e da Comissão de Ética, no caso daqueles que assim o entenderam necessário) e a colaboração dos médicos de 8 deles, havendo assim 89 respondentes. Segundo a Administração Central do Sistema de Saúde, I.P., na Região Norte de Portugal encontravam-se, em 2011, a exercer funções no Serviço Nacional de Nacional de Saúde (SNS) 413 médicos pediatras e 104 internos de Pediatria (Fonte: ACSS, 2011); assim, a amostra em estudo representa $17.2 \%$ dos pediatras e internos de pediatria do local e época em estudo.

Recolhidos os inquéritos, procedeu-se à sua análise estatística (descritiva, de referência cruzada e com testes de correlação Qui-quadrado para um nível de significância de 0.05), utilizando-se o software SPSS Statistics 20.

\section{RESULTADOS}

A maioria dos 89 respondentes era do sexo feminino $(\mathrm{n}=68 ; 76.4 \%)$ e a idade média foi de 39 anos $(\min =24 ; \max =61 ; \mathrm{SD}=10,8)$. Destes, a média de tempo de trabalho exercida na área da pediatria era de 13.5 anos $(\min =1$; $\max =38 ; \mathrm{SD}=11$ ), sendo que os médicos especialistas representaram $60.2 \%$ da amostra $(n=53)$. 


\section{Deteção do abuso}

A quase totalidade dos médicos consideraram ser sua responsabilidade detetar os casos de abuso nas crianças e adolescentes $(96.6 \%)$. A maioria referiu sentir-se quase sempre apta a detetar abuso físico $(\mathrm{n}=68 ; 76.4 \%)$, mas muitos consideraram-se pouco capazes de detetar o abuso sexual $(\mathrm{n}=44 ; 49.4 \%)$ e o psicológico $(n=41 ; 46.1 \%)$. As dificuldades mais frequentemente indicadas para a deteção dos abusos foram o diagnóstico diferencial da etiologia de uma lesão $(42 \%)$ e a inespecificidade dos sintomas (74\%); note-se que $23.9 \%$ dos médicos considerou ser raro o recurso destas vítimas ao SNS (Quadro I). Não se verificou existir correlação entre estas dificuldades sentidas e a idade ( $p=0.550 ; p=0.892)$, sexo $(p=0.932 ; p=0.669)$ ou anos de serviço $(p=0.854$; $p=0.988$ ) dos respondentes nos testes de independência efetuados.

Constatou-se que $29.2 \%$ dos médicos $(\mathrm{n}=26)$ não detetou nenhum caso de abuso no último ano, obtendo-se um total de 257 deteções (uma média de 3 deteções por médico e por ano).

\section{Quadro I - Dificuldades mais sentidas pelos médicos na deteção dos abusos}

\begin{tabular}{lr}
\hline & $\mathrm{n}(\%)$ \\
\hline Falta de formação específica durante o curso de Medicina & $17(19.3)$ \\
Falta de formação específica durante a especialização & $23(26.1)$ \\
Dificuldades no diagnóstico diferencial da etiologia das lesões & $37(42.0)$ \\
Inespecificidade dos sintomas & $66(75.0)$ \\
Constrangimento perante os cuidadores & $12(13.6)$ \\
Raridade do recurso destas crianças ao SNS & $21(23.9)$ \\
\hline
\end{tabular}

\section{Sinalização e Denúncia}

Todos os inquiridos consideraram ser responsabilidade sua sinalizar os casos de abuso com que contatam no exercício da atividade profissional mas $6.7 \%(\mathrm{n}=6)$ achou que não estava obrigado a denunciar. Note-se, contudo, que $30.3 \%(\mathrm{n}=27)$ referiram que sinalizar e denunciar constituem o mesmo procedimento.

A maioria dos indivíduos $(\mathrm{n}=78 ; 82 \%)$ consideraram saber sinalizar e/ou denunciar mas, uma vez mais, 32.9\% destes referiram que a sinalização e a denúncia são o mesmo. Não se observou correlação estatística entre estas variáveis (Quadro II). 


\section{Quadro II - Saber denunciar e/ou sinalizar e saber distinguir entre sinalização e denúncia}

\begin{tabular}{ccccc} 
& & \multicolumn{3}{c}{ Saber distinguir entre sinalização e denúncia } \\
\cline { 3 - 5 } & & Sim & Não & Total \\
\hline \multirow{2}{*}{ Saber sinalizar } & Não & 13 & 3 & $16(18.0 \%)$ \\
\multirow{2}{*}{ e/ou denunciar } & Sim & 49 & 24 & $73(82.0 \%)$ \\
& Total & $62(69.7 \%)$ & $27(30.3 \%)$ & $89(100 \%)$ \\
\hline
\end{tabular}

Desta última amostra (n=78), 52.1\% não considerou ser graças à instrução académica e profissionalizante que está apto a sinalizar e/ou denunciar. Por outro lado, dos $47.2 \%$ respondentes que sentem ter tido formação insuficiente, apenas 9.5\% admitiram não saber fazê-lo (Quadro III); encontrou-se aqui uma correlação inversa (coeficiente $=-0.208$ ) estatisticamente significativa $(p=0.05)$, ou seja, saber sinalizar e/ou denunciar surge associado ao facto de não terem tido formação específica nesta matéria.

\section{Quadro III - Saber sinalizar e/ou denunciar e instrução académica e profissionalizante na área}

\begin{tabular}{lcccc} 
& & \multicolumn{3}{c}{ Instrução na matéria } \\
\cline { 3 - 5 } & & Não & Sim & Total \\
\hline \multirow{2}{*}{ Saber sinalizar } & Não & 4 & 12 & $16(18.0 \%)$ \\
e/ou denunciar & Sim & 38 & 35 & $73(82.0 \%)$ \\
\cline { 3 - 5 } & Total & $42(47.2 \%)$ & $47(52.8 \%)$ & $89(100 \%)$ \\
\hline
\end{tabular}

Dos 63 respondentes que referem ter detetado pelo menos um caso no último ano, a maioria (79.4\%) disse ter sinalizado e/ou denunciado todos eles. É de notar que $7.9 \%$ sinalizou e/ou denunciou menos de metade dos casos mas $80 \%$ destes tinha referido saber fazê-lo; $4.8 \%$ admitiu não ter sinalizado e/ou denunciado nenhum caso, apesar de $66.7 \%$ destes referirem saber fazê-lo (Quadro IV); não se verificou existir qualquer correlação estatística entre estas variáveis $(p=0.815)$. Dois médicos $(3.2 \%)$ admitiram ter deixado casos por sinalizar e/ou denunciar a pedido da vítima.

Quadro IV - Saber sinalizar e/ou denunciar e número de casos comunicados

\begin{tabular}{lcccccc}
\cline { 3 - 6 } & & \multicolumn{4}{c}{$\mathrm{N}^{\circ}$ de casos sinalizados e/ou denunciados } & \\
\cline { 3 - 6 } & & Nenhum & $<50 \%$ & $\geq 50 \%$ & Todos & Total \\
\hline \multirow{2}{*}{ Saber sinalizar } & Não & 1 & 1 & 1 & 7 & $10(15,9 \%)$ \\
\multirow{2}{*}{ e/ou denunciar } & Sim & 2 & 4 & 4 & 43 & $53(84,1 \%)$ \\
& Total & $3(4,8 \%)$ & $5(7,9 \%)$ & $5(7,9 \%)$ & $50(79,4 \%)$ & $63(100 \%)$ \\
\hline
\end{tabular}

Foram $23.8 \%$ os que referiram já ter contatado com casos de abuso e consideraram não produtiva a sua articulação com o NHACJR, nomeadamente 
2 dos 3 respondentes que não sinalizaram e/ou denunciaram nenhum dos casos que acompanharam (Quadro V). Não há, no entanto, uma correlação estatística entre estas variáveis $(p=0.20)$. Consideraram importante a cooperação da especialidade de Medicina Geral e Familiar no acompanhamento de um caso de abuso a criança ou adolescente $94.4 \%$ dos médicos de pediatria. Admitiram ser vantajosa a implementação de novos protocolos de atuação para a abordagem dos casos de abuso 95.5\% dos médicos, sem que isso se correlacione com o facto de se considerarem ou não aptos a sinalizar e/ou denunciar os casos ( $p=0.344)$, ou o facto de conseguirem, ou não, uma ajuda importante por parte do NHACJR $(p=0.276)$.

Quadro V - Articulação com o NHACJR e número de casos comunicados

Número de casos sinalizados e/ou denunciados

\begin{tabular}{rcccccc} 
& & Nenhum & $<50 \%$ & $\geq 50 \%$ & Todos & Total \\
\hline \multirow{2}{*}{ NHACJR } & Sim & 2 & 0 & 1 & 12 & $15(23,8 \%)$ \\
\cline { 3 - 7 } & Não & 1 & 5 & 4 & 38 & $48(76,2 \%)$ \\
\cline { 3 - 7 } & Total & $3(4,8 \%)$ & $5(7,9 \%)$ & $5(7,9 \%)$ & $50(79,4 \%)$ & $63(100 \%)$ \\
\hline
\end{tabular}

\section{Perfil do médico}

Finalmente procurou-se traçar o perfil do médico (quanto à sua idade e sexo) face aos aspetos abordados (Quadro VI). Encontrou-se uma correlação estatisticamente significativa entre a idade e o facto de estes considerarem que sabem sinalizar e/ou denunciar $(p<0.001)$ e uma correlação inversa (coeficiente $=-0.273$ ) entre a sua idade e a perceção da necessidade de implementação de novos protocolos, ou seja, foram os médicos mais jovens a admitir não saber sinalizar e/ou denunciar e a referir a necessidade de novos protocolos de atuação.

Quadro VI - Correlações $(p)$ entre o perfil do respondente e as atitudes face ao abuso

\begin{tabular}{lccccc} 
& $\begin{array}{c}\text { Dever de } \\
\text { deteção }\end{array}$ & $\begin{array}{c}\text { Saber sinalizar e/ou } \\
\text { denunciar }\end{array}$ & $\begin{array}{c}\text { Obrigação de } \\
\text { denunciar }\end{array}$ & Instrução & $\begin{array}{c}\text { Implementação de } \\
\text { protocolos }\end{array}$ \\
\hline Idade & 0.591 & $<0.001$ & 0.103 & 0.447 & $0.01^{*}$ \\
\hline Sexo & 0.333 & 0.073 & 0.683 & 0.591 & 0.208 \\
\hline
\end{tabular}

*coeficiente de correlação=-0.273 


\section{DISCUSSÃO}

A literatura sobre este assunto, apesar da sua relevância, não é muito abundante, importando registar um trabalho Português que, não se debruçando exatamente sobre a matéria aqui analisada, aborda a temática da intervenção dos pediatras nestes casos ${ }^{9}$. Por outro lado, os estudos estrangeiros sobre a triagem dos abusos pelos pediatras, não incidem concretamente sobre a questão da sinalização ou denúncia da forma que aqui a perspetivamos, dado que estes aspetos são específicos do contexto legal de cada país. Assinale-se, contudo, que na maior parte dos países desenvolvidos, designadamente nos EUA, a questão da deteção e comunicação destes casos está na ordem do dia, sendo os médicos "mandatory reporters".

Não se conhecem, em Portugal, os valores reais da frequência com que são vítimas de abuso as crianças e adolescentes. No primeiro semestre de 2011 foram registadas pelas Forças de Segurança apenas 943 participações relativas a vítimas até aos 18 anos $^{10}$. Em 2010, foram instaurados pelas CPCJ 8550 processos de negligência, 441 de abandono, 3683 de abuso emocional, 1847 de abuso físico, 548 de abuso sexual e 26 de exploração do trabalho infantil $^{11}$. A Delegação do Norte do INMLCF observou entre 2010 e 2011, um total de 773 casos de alegados casos de abuso físico e 123 de abuso sexual em contexto familiar (Fonte: INMLCF), refletindo seguramente estes números, não só a falta de deteção e sinalização/denúncia mas, também, mostrando que muitas vítimas não são referenciadas para perícias médico-legais, designadamente nos casos de negligência e abuso psicológico.

No presente estudo, a quantidade de sinalizações e/ou denúncias (cerca de 200 , tendo em conta o total de 257 deteções e os $79.4 \%$ dos médicos que afirmam ter sinalizado e/ou denunciado todos os casos) parece estar minimamente em sintonia com os dados referidos pelas CPCJ e pelo INMLCF, atendendo à representatividade desta amostra e ao facto de os médicos constituírem apenas uma parte das entidades sinalizadoras dos processos. No entanto, $29.2 \%$ dos médicos referiram não ter detetado nenhum caso, o que pode significar, com elevada probabilidade, que, face a casos concretos, não foram capazes de os identificar. Reitera-se, contudo, que estes são os profissionais mais importantes na deteção destes casos, sobretudo na idade pré-escolar, devendo por isso ser capazes de os identificar e comunicar devidamente, de forma a possibilitar a implementação das devidas medidas de proteção e a eventual investigação criminal.

Apenas 52.8\% da amostra considerou satisfatória a instrução recebida no âmbito da Violência Doméstica e Maus-Tratos, e grande parte admitiu ter dificuldades na deteção do abuso sexual e psicológico. Num estudo dos EUA sobre a preparação facultada aos médicos internos para o rastreio de abuso 
sobre crianças, cerca de $80 \%$ afirmou que recebeu pouco treino nesta área e por isso não rastreiam os abusos como deveriam, com apenas $21 \%$ a fazê-lo ${ }^{12}$. Noutro estudo, 74\% dos pediatras e médicos de Medicina Geral e Familiar de um determinado hospital, referiu não ter recebido formação específica em abusos (sobretudo os pediatras), apresentando resultados menos satisfatórios que os médicos formados noutro hospital onde era obrigatório este tipo de instrução ${ }^{13}$. Em novembro de 2009, o American Board of Pediatrics realizou o primeiro exame de certificação de uma nova subespecialidade pediátrica: Pediatria do Abuso de Crianças ${ }^{14}$. Este é um passo fundamental, dado que a complexidade do assunto em causa não se compadece com a intervenção por profissionais que - apesar de interessados e bem-intencionados - não têm formação especializada no assunto.

Por outro lado, importa que os médicos saibam que é obrigatória a realização de uma perícia médico-legal sempre que se vislumbre a possibilidade de existirem danos corporais ou vestígios (artigos $151^{\circ}$ e $159^{\circ}$ do Código de Processo Penal), a qual não deve ser protelada pela intervenção de outros médicos (excetuando tratamentos urgentes), para evitar a vitimização secundária e a contaminação de vestígios e dos relatos. Para garantir a boa articulação destes procedimentos, celebrou-se em 2011 o protocolo atrás referido; este fixa os termos da colaboração entre médicos do SNS e do $\mathrm{INMLCF}^{8}$. As normas já publicadas pelo INMLCF para a realização dos exames forenses nestes $\operatorname{casos}^{15}$ não deverão ser consideradas suficientes para colmatar a falta de formação referida por vários pediatras, designadamente quando, à luz daquele protocolo, têm de ser estes a realizar o exame físico para colheita e preservação de vestígios, sendo fundamental formação específica nesta matéria.

Sinalização e denúncia devem complementar-se para garantir a melhor abordagem num caso de abuso. Como regulamentado pela DGS, a intervenção pretende-se executada nas formas de investigação criminal e de promoção e proteção da vítima, encontrando-se esta última organizada em três níveis de responsabilidade. No primeiro, as entidades competentes são os serviços de saúde, educação e ocupação dos tempos livres, encontrando-se os especialistas de Pediatra, de Medicina Geral e Familiar ou os Ginecologistas numa posição privilegiada detetar o abuso. Aos NACJR e NHACJR cabe um papel de consultoria ou até de condução do processo ${ }^{16}$. Não se conseguindo remover o perigo, torna-se de crucial importância a sinalização da vítima, sendo este o ato de dar conhecimento de uma situação ou suspeita de abuso, mediante comunicação às entidades competentes - as CPCJ da sua área de residência ${ }^{17}$. O último recurso será o Tribunal de Família e Menores, nível este que se pretende residual. Neste sentido, a DGS atualizou em 2011 o documento técnico de orientação da atuação médica perante casos de abuso sobre a criança e adolescente. Nele constam algoritmos - para a deteção e 
intervenção perante fatores de risco de abuso, sinais de alerta, abuso evidente e situações de perigo - e modelos de fichas de sinalização e denúncia. Frisa-se, também, que a "denúncia não tem tempo limite para ser apresentada e deverá acontecer depois de se ponderar e assegurar que a proteção da criança/jovem não será posta em causa por tal diligência"16. Note-se, contudo, que esta nota deve ser considerada com muito cuidado, primeiro porque a violência doméstica e os maus-tratos, à luz da legislação Portuguesa, têm de ser obrigatoriamente denunciados no mais curto espaço de tempo, segundo, porque como infelizmente a prática tem provado, em alguns casos em que a denúncia não é feita, não havendo uma intervenção formal, o caso pode escapar-se das mãos dos profissionais que o acompanham, havendo por vezes desfechos fatais. De facto, este importante documento, enferma de algumas lacunas do ponto de vista da intervenção criminal.

Mesmo julgando saber sinalizar e/ou denunciar, 4.8\% dos médicos admitiram não o ter feito em nenhum caso e $7.9 \%$ apenas o fizeram em menos de metade dos casos, não se correlacionando isso com deficiências na articulação com os NHACJR. De facto, é sabido que grande parte dos abusos não são comunicados ${ }^{9}$. Também em matéria de sinalização e denúncia, para estes médicos a instrução prévia não foi produtiva e o que se comprova é que muitos não conhecem a dinâmica processual da investigação criminal e da promoção e proteção, ao desconhecerem a diferença entre sinalização e denúncia, bem como a sua obrigação legal de o fazer. Verificou-se que são os médicos mais jovens os que mais admitem estas dificuldades, sendo recetivos à implementação de protocolos de atuação. Alguns médicos não se consideram obrigados a denunciar (6.7\%) mas, embora esta a lei seja relativamente recente, tal não se correlacionou com a idade ou anos de serviço dos médicos. Entre outras razões que levam os médicos a não efetuar a denúncia, podem

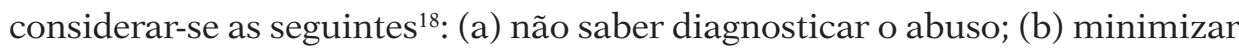
a gravidade dos indicadores pelo contexto cultural; (c) não aceitar isso como uma obrigação profissional sua, defendendo-se através do sigilo médico; (d) desconhecimento da atitude a tomar; (e) medo de represálias contra si ou os seus; (f) querer evitar acusações de falsa denúncia, a atenção da imprensa e o comparecimento em tribunal.

A American Academy of Pediatrics enfatiza que identificar uma situação de abuso exercida sobre um seu cuidador pode prevenir esta forma de violência sobre a criança ${ }^{19}$. Um estudo acrescenta que muitas mulheres vítimas de abuso se revelaram ao pediatra dos filhos ${ }^{20}$. A deteção e sinalização/denúncia destes casos de exposição das crianças à violência nas relações de intimidade já é feita por médicos do INMLCF, com resultados benéficos, dado que tal constitui uma forma grave de abuso. 


\section{CONCLUSÃO}

$\mathrm{Na}$ amostra estudada, verificou-se existirem ainda muitas lacunas de conhecimento por parte dos médicos da área de pediatria no que se refere á sua capacidade de detetarem e comunicarem os casos de abuso. Assim, dada à relevância do contributo destes profissionais para a identificação destes casos, é importante desenvolver estratégias de sensibilização para o rastreio ativo dos mesmos e de articulação entre os diversos profissionais e instituições envolvidas, dado que a intervenção nestes casos é obrigatoriamente multidisciplinar. Igualmente parece ser fundamental a formação específica destes médicos nesta matéria.

\section{REFERÊNCIAS BIBLIOGRÁFICAS}

1 Magalhães T. Violência e Abuso. Respostas Simples para Questões Complexas. Coimbra: Imprensa da Universidade de Coimbra; 2010.

2 Dias I, Ribeiro CS, Magalhães T. A construção social do abuso na infância. In: Magalhães T, editor. Abuso de Crianças e Jovens. Da Suspeita ao Diagnóstico. Lisboa: Lidel; 2010. 8-9.

3 Magalhães T, Jardim P, Santos L, Pinto NF, Oliveira RJD, Caldas IM. Indicadores físicos de abuso. In: Magalhães T, editor. Abuso de Crianças e Jovens. Da Suspeita ao Diagnóstico. Lisboa: Lidel; 2010. 51-56.

4 Pires ALD, Miyazaki MCOS. Maus-tratos contra crianças e adolescentes: revisão da literatura para profissionais da saúde. Arq Ciênc Saúde 2005 jan-mar; 12(1):42-49.

5 Jardim P, Magalhães T. Indicadores físicos e biológicos do abuso sexual. In: Magalhães T, editor. Abuso de Crianças e Jovens. Da Suspeita ao Diagnóstico. Lisboa: Lidel; 2010. 109-111

6 Cunningham A, Hurley P. A Full and Candid Account. Children \& Teenagers Testifying in Domestic Violence Cases. London (Canadá): Centre for Children and Families in the Justice System (London Family Court Clinic, Inc.); 2007.

7 Stiles M. Witnessing Domestic Violence: The Effect on Children. Am Fam Physician 2002;66(11): 2052-2067.

8 Magalhães T, Vieira DN. Proteção de crianças e jovens em risco: considerações sobre o protocolo nacional de 2011. Revista Portuguesa do Dano Corporal 2011; 22: 9-22.

9 Vasconcelos A, Cardoso BM, Barros M, Almeida H. Abordagem da criança vítima de maus tratos na Urgência Pediátrica - 5 anos de experiência do Hospital Prof. Doutor Fernando Fonseca. Acta Pediatr Port 2011;42(1):8-11.

10 Direcção Geral da Administração Interna. Relatório de monitorização da Violência Doméstica - $1^{\circ}$ Semestre de 2011. Lisboa: Ministério da Administração Interna, Direcção-Geral de Administração Interna Direcção de Serviços de Planeamento Estratégico, Núcleo de Estudos e Análise Prospectiva em Segurança Interna (NEAPSI); 2011. 
11 Carvalho R (coord). Relatório Anual de Avaliação da Atividade das Comissões de Proteção de Crianças e Jovens no ano de 2010. Lisboa: CNPCJR; 2011.

12 Bair-Merritt MH, Giardino AP, Turner M, Ganetsky M, Christian CW. Pediatric residency training on domestic violence: a national survey. A 2004;4(1): 24-7. PubMed 2004.

13 Erickson MJ, Hill TD, Siegel RM. Barriers to Domestic Violence Screening in the Pediatric Setting. NeoReviews 2001;108(1): 98-102

14 Block RW. Child abuse requires specialized treatment, reporting. Amednews.com 2010 [cited 2012 May 1]. Acessível em http://www.ama-assn.org/amednews/2010/06/28/ prca0628.htm.

15 Magalhães T, Ribeiro CS, Jardim P. Procedimentos Forenses No Âmbito da Recolha de Informação, Exame Físico e Colheita de Vestígios em Crianças e Jovens Vítimas de Abuso Físico e/ou Sexual. Acta Med Port 2011; 24: 339-348.

16 Leça A et al. Maus Tratos em Crianças e Jovens - Guia prático de abordagem, diagnostico e intervenção. Direcção-Geral da Saúde; 2011.

17 Pinto da Costa D, Taborda MJ, Magalhães T. Da suspeita ou deteção à sinalização do abuso. In: Magalhães T, editor. Abuso de Crianças e Jovens. Da Suspeita ao Diagnóstico. Lisboa: Lidel; 2010. 139-146.

18 Cury M (coord) Estatuto da Criança e Adolescente Comentado: comentários jurídicos e sociais. São Paulo: Malheiros;1992. 737-8.

19 American Academy of Pediatrics, Committee on Child Abuse and Neglect: The Role of the Pediatrician in Recognizing and Intervening on Behalf of Abused Women. Pediatrics 1998; 101(6): 1091 -1092.

20 Siegel RM, Hill TD, Henderson VA, Ernst HM, Boat BW. Screening for domestic violence in the community pediatric setting. Pediatrics 1999; 104(4 Pt 1): 874-7. Pubmed 1999.

Resumo: Abuso contra crianças. A perceção do médico na intervenção pediátrica

0 abuso constitui, além de uma questão legal e social, um problema grave e frequente de saúde. No caso das crianças vítimas, o papel dos serviços de saúde, designadamente ao nível da Pediatria, revela-se fundamental na sua deteção, sinalização e/ou denúncia, para além do papel ao nível do seu tratamento e acompanhamento. Assim, o objetivo deste trabalho é analisar as perceções dos médicos que trabalham na área da Pediatria relativamente à sua capacidade para intervirem nesta matéria. Para tal, efetuou-se um inquérito a 89 pediatras ou internos de pediatria. Constatou-se que muitos médicos (76.4\%) sentem ter quase sempre capacidade para detetar o abuso físico, mas $49.4 \%$ e $46.1 \%$, considerou-se, respetivamente, pouco capaz de detetar abuso sexual ou o abuso psicológico. Os conceitos de sinalização e denúncia são confundidos por $30.3 \%$ dos médicos e $6.7 \%$ ainda desconhece a obrigação de denunciar estes casos. Só $52.8 \%$ acha satisfatória a instrução académica e profissionalizante que teve nesta área. Ainda se verificam, pois, muitas lacunas na preparação dos médicos da área da Pediatria para a abordagem destes casos, havendo necessidade de mais formação, melhor divulgação das medidas já implementadas e de criação de novos protocolos, mais adaptados e específicos à realidade de cada comunidade.

Palavras-Chave: Abuso; criança; deteção; comunicação. 
Summary: Abuse against children. The physician's perception in the paediatric intervention The abuse is not only a legal and social issue, but also a serious and frequent health problem. In the case of children, the role of health care professionals, particularly in paediatrics' units, is fundamental for its detection and report, as well as for treatment and follow-up. Thus, the objective of this study is to analyze the perceptions of physicians working in the field of paediatrics' units concerning their ability to intervene in this issue. On a survey to 89 physicians, working in hospital paediatric departments, several problems concerning the efficacy of these processes were found. Many physicians (76.4\%) declared being almost always able to detect physical abuse, but they affirmed to be less able to do so on sexual (49.4\%) and psychological (46.1\%) abuse. The concepts of referral and denouncement are misunderstood by $30.3 \%$ of them and $6.7 \%$ don't know their duty of reporting. Only $52.8 \%$ consider satisfactory the academic and professionalising training on these issues. There are still many flaws on the preparation of the professionals to deal with these cases. More training, better divulgation of the existing measures and the creation of new and more specific protocols are needed.

Key-Words: Abuse; child; detection; report.

Pedido de separatas:

TERESA MAGALHÃES

imlfmup@gmail.com 